\title{
TORSION CLASSES, WIDE SUBCATEGORIES AND LOCALISATIONS
}

\author{
FREDERIK MARKS, JAN ŠŤOVÍČEK
}

\begin{abstract}
For a finite dimensional algebra $A$, we establish correspondences between torsion classes and wide subcategories in $\bmod (A)$. In case $A$ is representation finite, we obtain an explicit bijection between these two classes of subcategories. Moreover, we translate our results to the language of ring epimorphisms and universal localisations. It turns out that universal localisations over representation finite algebras are classified by torsion classes and support $\tau$-tilting modules.
\end{abstract}

\section{INTRODUCTION}

Torsion classes and wide (i.e abelian and extension closed) subcategories of a given module category play an important role in the representation theory of rings and algebras. In this article, we show that these two classes of subcategories are intrinsically connected. If $A$ is a finite dimensional algebra, we obtain an injection from wide subcategories to torsion classes in $\bmod (A)$. When restricting to functorially finite subcategories this map can be turned around yielding an injection from functorially finite torsion classes to functorially finite wide subcategories. Consequently, over representation finite algebras torsion classes are in bijection with wide subcategories.

We use this bijection to classify all universal localisations in the sense of $[\mathrm{Sch}]$. These localisations turn out to correspond to torsion classes and also to support $\tau$-tilting modules recently introduced in [AIR]. This connection not only allows us to readily learn basic parameters of the corresponding localisations (such as the number of simple modules over the localised ring), but also opens the possibility to study the still somewhat mysterious universal localisations using advanced tools of representation theory. For instance, [AIR] introduces a well behaved mutation procedure for support $\tau$-tilting modules and it might be interesting to understand its counterpart for universal localisations.

Looking back at the concepts involved, the notion of a torsion class in an abelian category goes back to Dickson $([\mathrm{D}])$. These classes occur as the left-hand-side of a pair of subcategories $(\mathcal{T}, \mathcal{F})$ where $\mathcal{T}$ and $\mathcal{F}$ are chosen maximally with respect to the property that $\operatorname{Hom}(T, F)=0$ for all $T \in \mathcal{T}$ and $F \in \mathcal{F}$. When passing to module categories, torsion classes are studied, for example, in tilting theory (see $[\mathrm{AHK}]$ ). In fact, the subcategory of all modules generated by a tilting module forms a torsion class. Moreover, torsion classes are relevant to decide if a given subcategory of a module category has almost split sequences ([AS2]). Building on this work, it was shown in [AIR] that functorially finite torsion classes in $\bmod (A)$ are parametrised by support $\tau$-tilting modules.

Wide subcategories of a given module category are full abelian subcategories that are closed under extensions. In $[[\mathrm{H}]$, Hovey classified the wide subcategories of finitely presented modules over some commutative rings by certain subsets of the spectrum of the ring. This classification

2010 Mathematics Subject Classification. 16G20, 16S85, 18E40.

Key words and phrases. Finite dimensional algebra, torsion class, wide subcategory, universal localisation.

The second named author was supported by grant GA ČR 14-15479S from the Czech Science Foundation. 
can be understood as a module theoretical interpretation of the correspondence between thick subcategories of perfect complexes in the derived category and certain unions of closed sets of the spectrum (see [Hop, $\mathrm{N}, \mathrm{T}]$ ). Passing to non-commutative rings, it was shown in [IT] that over a finite dimensional hereditary algebra $A$, the functorially finite wide subcategories of $\bmod (A)$ are in bijection with the functorially finite torsion classes of the same category.

Universal localisations, as introduced by Cohn and Schofield (see $[\mathbf{S c h}]$ ), generalise classical localisation theory to possibly non-commutative rings. Instead of inverting a given set of elements, one localises with respect to a set of maps between finitely generated projective modules. Although universal localisations were already successfully used in different areas of mathematics like topol$\operatorname{ogy}([\mathbb{R}])$, algebraic K-theory $([\mathrm{NR}])$ and tilting theory $([\overline{\mathrm{AA}}])$, the concept itself is not yet fully understood. In our context, universal localisations turn out to be relevant, since the modules over the localised ring always form a wide subcategory of the initial module category.

In this article, we work over an arbitrary finite dimensional algebra $A$ over a field and we are interested in the category $\bmod (A)$ of finite dimensional $A$-modules. We start by associating a torsion class to every wide subcategory and we show that this assignment is injective (Proposition 3.3). However, in general, there are more torsion classes than wide subcategories (Example 3.4). In a second step, we restrict the setting to functorially finite subcategories and we obtain an injection in the other direction - from functorially finite torsion classes to functorially finite wide subcategories (Proposition 3.9). We also give sufficient conditions for this map to be bijective (Theorem 3.10 and Corollary 3.11). In a final section, we discuss applications of our results to ring epimorphisms and universal localisations. In fact, classifying functorially finite wide subcategories in $\bmod (A)$ amounts to classifying certain epimorphisms of rings starting in $A$. We provide sufficient conditions for these ring epimorphisms to be universal localisations (Proposition 4.4). Moreover, we show that for representation finite algebras torsion classes and wide subcategories are in bijection with universal localisations (see Theorem 4.5).

\section{NOTATION}

Throughout, let $A$ be a finite dimensional algebra over an algebraically closed field $\mathbb{K}$. The category of all finitely generated left $A$-modules will be denoted by $\bmod (A)$. All subcategories $C$ of $\bmod (A)$ are considered to be full and replete. A subcategory $\mathcal{C}$ of $\bmod (A)$ is called functorially finite, if every $A$-module admits both a left and a right $C$-approximation. More precisely, for every $X \in \bmod (A)$ we need objects $C_{1}, C_{2} \in \mathcal{C}$ and morphisms $g_{1}: X \rightarrow C_{1}$ and $g_{2}: C_{2} \rightarrow X$ such that the maps $\operatorname{Hom}_{A}\left(g_{1}, \tilde{C}\right)$ and $\operatorname{Hom}_{A}\left(\tilde{C}, g_{2}\right)$ are surjective for all $\tilde{C} \in \mathcal{C}$. Moreover, a subcategory $C$ is called wide, if it is exact abelian and closed for extensions and it is called a torsion class, if it is closed for quotients and extensions. By wide $(A)$ (respectively, $f$-wide $(A)$ ) we denote the class of all (functorially finite) wide subcategories in $\bmod (A)$. Moreover, by $\operatorname{tor}(A)$ (respectively, $f$-tors $(A)$ ) we denote the class of all (functorially finite) torsion classes in $\bmod (A)$. For a module $X$ in $\bmod (A)$, we denote by $\operatorname{add}(X)$ the subcategory of $\bmod (A)$ containing all direct summands of finite direct sums of copies of $X$. For a given subcategory $\mathcal{C}$, gen $(\mathcal{C})$ describes the subcategory containing all quotients of finite direct sums of objects from $\mathcal{C}$ and the subcategory filt $(\mathcal{C})$ is built from all finitely generated $A$-modules $X$ admitting a finite filtration of the form

$$
0=F_{0} \subseteq F_{1} \subseteq \underset{2}{2} F_{2} \subseteq \ldots \subseteq F_{n}=X
$$


with $F_{i} / F_{i-1} \in \mathcal{C}$ for all $1 \leq i \leq n$ and $n \in \mathbb{N}$. We say that the module $X$ possesses a $\mathcal{C}$-filtration of length $n$ and the minimal length of such a filtration is called the $\mathcal{C}$-length of $X$. Finally, note that $\operatorname{filt}(\mathcal{C})$ is the smallest subcategory of $\bmod (A)$ containing $\mathcal{C}$ and being closed under extensions.

\section{TORSION CLASSES AND WIDE SUBCATEGORIES}

We start by associating a torsion class to every wide subcategory $\mathcal{W}$ in wide $(A)$. We set

$$
\mathcal{T}_{\mathcal{W}}:=\operatorname{filt}(\operatorname{gen}(\mathcal{W}))
$$

Lemma 3.1. $\mathcal{T}_{\mathcal{W}}$ is a torsion class in $\bmod (A)$.

Proof. By construction, it suffices to check closure for quotients. Take $X$ in $\mathcal{T}_{\mathcal{W}}$ and a surjection $X \rightarrow X^{\prime}$ in $\bmod (A)$. We show by induction on the $\operatorname{gen}(\mathcal{W})$-length of $X$ that also $X^{\prime}$ belongs to $\mathcal{T}_{\mathcal{W}}$. Clearly, if $X$ lies in $\operatorname{gen}(\mathcal{W}) \subseteq \mathcal{T}_{\mathcal{W}}$, then so does $X^{\prime}$. For the general case, take a short exact sequence of the form

$$
0 \longrightarrow M_{1} \longrightarrow X \longrightarrow M_{2} \longrightarrow 0
$$

with $M_{1}$ in $\operatorname{gen}(\mathcal{W})$ and $M_{2}$ in $\mathcal{T}_{\mathcal{W}}$ of strictly smaller $g e n(\mathcal{W})$-length than $X$. We get the following induced commutative diagram

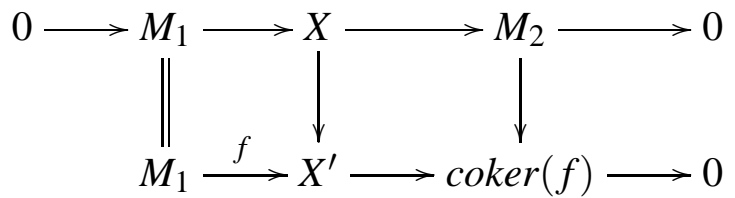

By induction hypothesis, $\operatorname{coker}(f) \in \mathcal{T}_{\mathcal{W}}$ and, thus, $X^{\prime} \in \mathcal{T}_{\mathcal{W}}$, as an extension of modules in $\mathcal{T}_{\mathcal{W}}$.

Lemma 3.2. The category $\mathcal{W}$ is closed for subobjects in $\mathcal{T}_{\mathcal{W}}$. More precisely, if $W \in \mathcal{W}$ and $X \subseteq W$ is a submodule that belongs to $\mathcal{T}_{\mathcal{W}}$, then $X \in \mathcal{W}$.

Proof. Take an object $W$ in $\mathcal{W}$ together with an injection $i: X \rightarrow W$ where $X$ belongs to $\mathcal{T}_{\mathcal{W}}$. We have to show that $X \in \mathcal{W}$. We proceed by induction on the $g e n(\mathcal{W})$-length of $X$. To begin with, if $X$ is in $\operatorname{gen}(\mathcal{W})$, then it already lies in $\mathcal{W}$ - as the image of a map in $\mathcal{W}$. For the general case, take a short exact sequence of the form

$$
0 \longrightarrow M_{1} \longrightarrow X \longrightarrow M_{2} \longrightarrow 0
$$

as in the proof of Lemma 3.1. We get the following induced commutative diagram

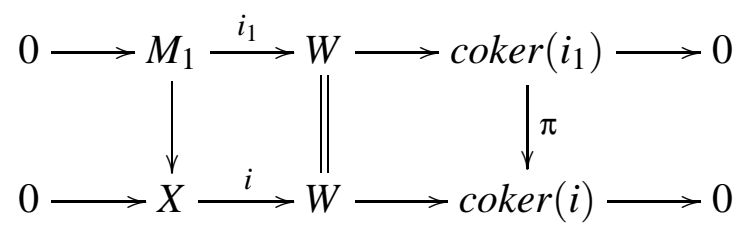

Since $M_{1}$ lies in $\operatorname{gen}(\mathcal{W})$ it already belongs to $\mathcal{W}$ and, thus, so does coker $\left(i_{1}\right)$. Moreover, by the Snake lemma, we have $M_{2} \cong \operatorname{ker}(\pi)$ and, hence, by induction hypothesis, also $M_{2}$ is in $\mathcal{W}$. Finally, since $\mathcal{W}$ is closed under extensions, one gets $X \in \mathcal{W}$ - as wanted. 
Next, we show how to recover the wide subcategory $\mathcal{W}$ from the torsion class $\mathcal{T}_{\mathcal{W}}$. We need the following construction due to [IT]. Let $\mathcal{T}$ be a torsion class. We define

$$
\alpha(\mathcal{T}):=\{X \in \mathcal{T} \mid \forall(g: Y \rightarrow X) \in \mathcal{T}, \operatorname{ker}(g) \in \mathcal{T}\}
$$

Following [IT, Proposition 2.12], $\alpha(\mathcal{T})$ is a wide subcategory of $\bmod (A)$. This result is actually an instance of a more general fact [KS, Exercise 8.23], since $\alpha(\mathcal{T})$ consists precisely of what is called $\mathcal{T}$-coherent objects in $[\mathrm{KS}]$.

Proposition 3.3. Let $\mathcal{W}$ be a wide subcategory of $\bmod (A)$. Then $\mathcal{W}=\alpha\left(\mathcal{T}_{\mathcal{W}}\right)$. In particular, mapping $\mathcal{W}$ to $\mathcal{T}_{\mathcal{W}}$ yields an injection from wide $(A)$ to tors $(A)$.

Proof. We start by showing that $\mathcal{W}$ is contained in $\alpha\left(\mathcal{T}_{\mathcal{W}}\right)$. Take $W$ in $\mathcal{W}$ and a test map $g: Y \rightarrow W$ with $Y$ in $\mathcal{T}_{\mathcal{W}}$. We have to show that $\operatorname{ker}(g)$ belongs to $\mathcal{T}_{\mathcal{W}}$. First, assume that $Y$ belongs to $\operatorname{gen}(\mathcal{W})$. Thus, there is some $W^{\prime}$ in $\mathcal{W}$ and a surjection $\pi$ yielding the following commutative diagram of $A$-modules

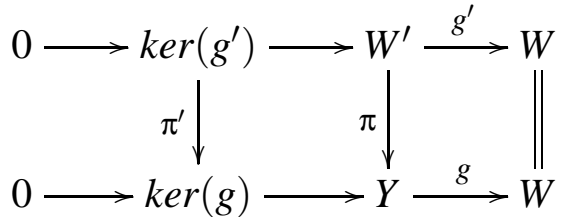

It follows that also the map $\pi^{\prime}$ must be surjective. Therefore, since $\operatorname{ker}\left(g^{\prime}\right)$ belongs to $\mathcal{W}$, the kernel of $g$ is in $\operatorname{gen}(\mathcal{W}) \subseteq \mathcal{T}_{\mathcal{W}}$ - as wanted. For the general case, take a short exact sequence

$$
0 \longrightarrow M_{1} \stackrel{i}{\longrightarrow} Y \longrightarrow M_{2} \longrightarrow 0
$$

with $M_{1}$ in $\operatorname{gen}(\mathcal{W})$ and $M_{2}$ in $\mathcal{T}_{\mathcal{W}}$ of strictly smaller $\operatorname{gen}(\mathcal{W})$-length than $Y$. We get the following induced commutative diagram of $A$-modules with exact columns

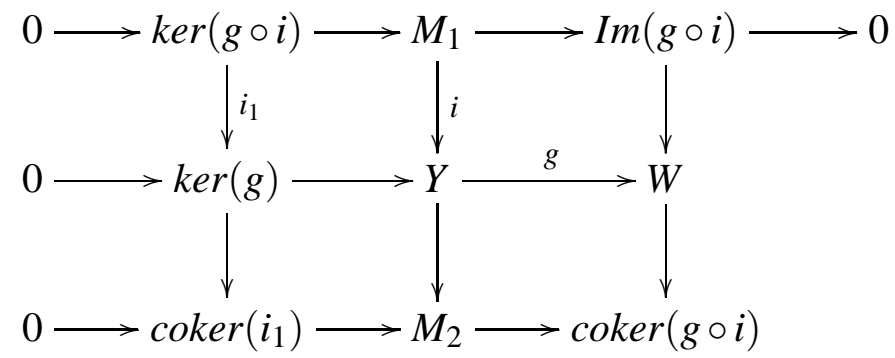

By Lemma 3.2, $\operatorname{Im}(g \circ i)$ is in $\mathcal{W}$ and, thus, so is $\operatorname{coker}(g \circ i)$. Now it follows from induction that $\operatorname{ker}(g \circ i)$ and $\operatorname{coker}\left(i_{1}\right)$ belong to $\mathcal{T}_{\mathcal{W}}$. Hence, since $\mathcal{T}_{\mathcal{W}}$ is closed for extensions, $\operatorname{ker}(g)$ is in $\mathcal{T}_{\mathcal{W}}$.

Next, we check that $\alpha\left(\mathcal{T}_{\mathcal{W}}\right) \subseteq \mathcal{W}$. Take a module $X$ in $\alpha\left(\mathcal{T}_{\mathcal{W}}\right)$. First, assume that $X$ belongs to $\operatorname{gen}(\mathcal{W})$. Thus, there is some $W \in \mathcal{W}$ and a surjection $\pi: W \rightarrow X$. By assumption, $K:=\operatorname{ker}(\pi)$ lies in $\mathcal{T}_{\mathcal{W}}$. Hence, by Lemma $3.2, K$ is in $\mathcal{W}$ and, since $\mathcal{W}$ is wide, $X \in \mathcal{W}$. For the general case, take a short exact sequence of the form

$$
0 \longrightarrow M_{1} \longrightarrow X \longrightarrow M_{2} \longrightarrow 0
$$

with $M_{1}$ in $\operatorname{gen}(\mathcal{W})$ and $M_{2}$ in $\mathcal{T}_{\mathcal{W}}$ of strictly smaller $\operatorname{gen}(\mathcal{W})$-length than $X$. Since, by definition, $\alpha\left(\mathcal{T}_{\mathcal{W}}\right)$ is closed for subobjects in $\mathcal{T}_{\mathcal{W}}$, it follows that also $M_{1}$ belongs to $\alpha\left(\mathcal{T}_{\mathcal{W}}\right)$ and, hence, so does $M_{2}$. Using induction, this already implies that $M_{1}$ and $M_{2}$ are in $\mathcal{W}$. In particular, $X$ belongs to $\mathcal{W}$ - as an extension of $M_{1}$ and $M_{2}$. This finishes the proof. 
The following example illustrates that, in general, for a given algebra $A$ there are more torsion classes than wide subcategories in $\bmod (A)$.

Example 3.4. Let $A$ be the Kronecker algebra defined as the path algebra over $\mathbb{K}$ of the quiver

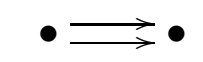

Let $\mathcal{T} \subseteq \bmod (A)$ be the torsion class given by all preinjective $A$-modules. It is not hard to check that $\alpha(\mathcal{T})=\{0\}$. In particular, there is no $\mathcal{W} \in$ wide $(A)$ such that $\mathcal{T}_{\mathcal{W}}=\mathcal{T}$.

Next, we restrict the setting to functorially finite torsion classes and functorially finite wide subcategories. First we recall important related definitions.

Definition 3.5 ([AMV, Definition 3.10]). Let $T \in \bmod (A)$ and $\sigma: P_{1} \rightarrow P_{0}$ be a projective presentation of $T$. Let us denote by $\mathcal{D}_{\sigma}$ the class

$$
\mathcal{D}_{\sigma}:=\left\{X \in \bmod (A) \mid \operatorname{Hom}_{A}(\sigma, X) \text { surjective }\right\} .
$$

We say that $T$ is silting with respect to $\sigma$ if $\mathcal{D}_{\sigma}=\operatorname{gen}(T)$. We say that $T$ is silting if there exists a projective presentation $\sigma$ of $T$ with respect to which $T$ is silting.

This concept was defined in [AMV] in the category of all modules, but if $T \in \bmod (A)$, our definition is easily seen to be equivalent. It was shown in [AMV, Proposition 3.16] that finite dimensional silting modules over $A$ coincide with support $\tau$-tilting modules from [AIR].

Now [AIR, Theorem 2.7] asserts that the functorially finite torsion classes $\mathcal{T} \subseteq \bmod (A)$ are parametrised by the isomorphism classes of basic silting modules, i.e. the silting modules $T$ with a decomposition $T=M_{1} \oplus \cdots \oplus M_{n}$ where all the $M_{i}$ are indecomposable and $M_{i} \not M_{j}$ for all $i \neq j$. Given $T$ silting, the corresponding functorially finite torsion class is simply $\mathcal{T}=\operatorname{gen}(T)$. If we conversely start with a torsion class $\mathcal{T}$, there is a $\mathcal{T}$-approximation sequence

$$
A \stackrel{\phi}{\longrightarrow} T_{0} \longrightarrow T_{1} \longrightarrow 0
$$

with $\phi$ left-minimal, i.e. for all $h \in \operatorname{End}_{A}\left(T_{0}\right), h \circ \phi=\phi$ implies that $h$ is an isomorphism. Now, the corresponding silting module is given by the direct sum of all non-isomorphic indecomposable direct summands of $T_{0}$ and $T_{1}$ (compare Lemma 3.7 below).

Definition 3.6. Let $C$ be a class of $A$-modules. A module $P \in C$ is Ext-projective in $C$ if we have $\operatorname{Ext}_{A}^{1}(P, M)=0$ for all $M \in C$. The module $P$ is split projective in $C$ if each surjective morphism $M \rightarrow P$ with $M \in C$ splits.

Lemma 3.7. Let $\mathcal{T} \subseteq \bmod (A)$ be a functorially finite torsion class and let

$$
A \stackrel{\phi}{\longrightarrow} T_{0} \longrightarrow T_{1} \longrightarrow 0
$$

be an exact sequence such that $\phi$ is a minimal left $\mathcal{T}$-approximation. Then add $\left(T_{0} \oplus T_{1}\right)$ is the class of all Ext-projective modules in $\mathcal{T}$. Moreover, an indecomposable module $P \in \operatorname{add}\left(T_{0} \oplus T_{1}\right)$ is split projective in $\mathcal{T}$ if and only if it is a summand of $T_{0}$.

Proof. We need to prove only the last sentence, the rest follows essentially from [AIR, §1]. Suppose that $P$ is an indecomposable summand of $T_{0} \oplus T_{1}$. If $P$ is split projective in $\mathcal{T}$, then, since $\mathcal{T}=\operatorname{gen}\left(T_{0}\right)$, there is a split epimorphism $T_{0}^{n} \rightarrow P$ and $P$ is a summand of $T_{0}$.

Suppose conversely that $P$ is a summand of $T_{0}$ and consider a surjection $p: X \rightarrow P$ with $X \in \mathcal{T}$. We must prove that $p$ splits. Since $\mathcal{T}=\operatorname{gen}\left(T_{0}\right)$, we can without loss of generality assume that 
$X \in \operatorname{add}\left(T_{0}\right)$. Moreover, $p$ splits if and only if $p \oplus 1_{T_{0} / P}: X \oplus T_{0} / P \rightarrow T_{0}$ splits. Hence, it suffices to prove that every surjection $p^{\prime}: X^{\prime} \rightarrow T_{0}$ with $X^{\prime} \in \operatorname{add}\left(T_{0}\right)$ splits. To this end, $\phi: A \rightarrow T_{0}$ factors as $\phi=p^{\prime} \circ f$ for some $f: A \rightarrow X^{\prime}$, since $p^{\prime}$ is surjective. On the other hand, since $X^{\prime} \in \operatorname{add}\left(T_{0}\right)$, there is a factorization $f=i \circ \phi$ for some $i: T_{0} \rightarrow X^{\prime}$ yielding the commutative diagram

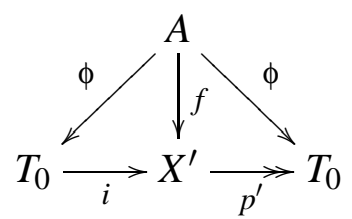

Putting this all together, we have $\phi=p^{\prime} \circ i \circ \phi$. Since $\phi$ is left-minimal, $p^{\prime}$ splits as required.

For an $A$-module $M$, we denote by $M^{\circ}$ the subcategory of $\bmod (A)$ given by all modules $X$ such that $\operatorname{Hom}_{A}(M, X)=0$. For a functorially finite torsion class $\mathcal{T}=\operatorname{gen}(T)$ in $\bmod (A)$ and $T_{1}$ chosen as above, we are interested in the intersection $\mathcal{W}_{\mathcal{T}}:=\mathcal{T} \cap T_{1}^{\circ}$. Note that, since $T_{1}$ is Ext-projective in $\mathcal{T}$, it follows that $\left(\operatorname{gen}\left(T_{1}\right), T_{1}^{\circ}\right)$ is a torsion pair in $\bmod (A)$. In particular, using the main theorem in $[\mathrm{Sm}], T_{1}^{\circ}$ is a functorially finite subcategory of $\bmod (A)$. Consequently, also $\mathcal{W}_{\mathcal{T}}$ is functorially finite. Indeed, we can construct left $\mathcal{W}_{\mathcal{T}}$-approximations by first taking a left $\mathcal{T}$-approximation and then factoring out the gen $\left(T_{1}\right)$-torsion part; right approximations are constructed dually (compare to the approach in [AMV2]).

Lemma 3.8. Let $\mathcal{T}=\operatorname{gen}(T)$ be a functorially finite torsion class in $\bmod (A)$. Then $\alpha(\mathcal{T})$ coincides with $\mathcal{W}_{\mathcal{T}}$. In particular, $\alpha(\mathcal{T})$ is functorially finite.

Proof. First, take $X$ in $\mathcal{W}_{\mathcal{T}}$ and consider a map $g: Y \rightarrow X$ in $\mathcal{T}$. We have to check that $\operatorname{ker}(g) \in \mathcal{T}$. Note that, by assumption, also $\operatorname{Im}(g)$ lies in $\mathcal{W}_{\mathcal{T}}$. Now we choose a surjection $\pi: A^{n} \rightarrow \operatorname{ker}(g)$ for some $n>0$. Using the left $\mathcal{T}$-approximation $\phi: A \rightarrow T_{0}$, we obtain the following commutative diagram of $A$-modules

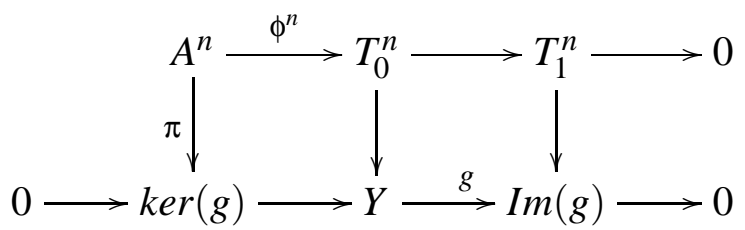

Since $\operatorname{Hom}_{A}\left(T_{1}, \operatorname{Im}(g)\right)=0$, there is a surjection $\tilde{\pi}: T_{0}^{n} \rightarrow \operatorname{ker}(g)$ such that $\pi=\tilde{\pi} \circ \phi^{n}$. Hence, $\operatorname{ker}(g)$ belongs to $\mathcal{T}$.

For the other inclusion, we take some $X$ in $\alpha(\mathcal{T})$. It suffices to show that $\operatorname{Hom}_{A}\left(T_{1}, X\right)=0$. Let $h: T_{1} \rightarrow X$ be such a map. We will prove that $\operatorname{Im}(h)$ is zero. First of all, observe that also $\operatorname{Im}(h)$ 
lies in $\alpha(\mathcal{T})$. Now consider the following induced commutative diagram of $A$-modules

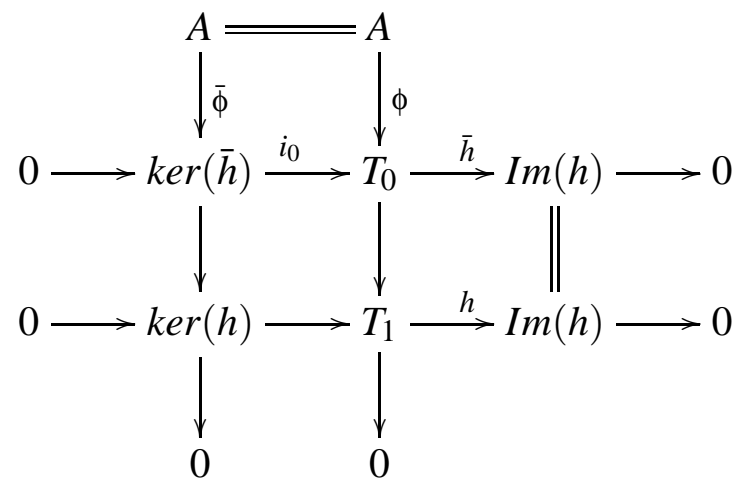

Since $\operatorname{Im}(h)$ belongs to $\alpha(\mathcal{T})$, the module $\operatorname{ker}(\bar{h})$ has to be in $\mathcal{T}$. Hence, using that $\phi$ is a left $\mathcal{T}$-approximation, there is a map $s: T_{0} \rightarrow \operatorname{ker}(\bar{h})$ with $s \circ \phi=\bar{\phi}$. It follows that $\phi=i_{0} \circ \bar{\phi}=i_{0} \circ s \circ \phi$. Since $\phi$ is left-minimal, $i_{0} \circ s$ has to be an isomorphism and, thus, so does $i_{0}$. In particular, $\operatorname{Im}(h)$ is zero, as wanted.

Now we are able to state the following key proposition in our context.

Proposition 3.9. Let $\mathcal{T}$ be in f-tors $(A)$. Then $\mathcal{T}=\mathcal{T}_{\alpha(\mathcal{T})}=$ filt $(\operatorname{gen}(\alpha(\mathcal{T})))$. In particular, mapping $\mathcal{T}$ to $\alpha(\mathcal{T})$ yields an injection from $f$-tors $(A)$ to $f$-wide $(A)$.

Proof. Since, by construction, $\mathcal{T}_{\alpha(\mathcal{T})}$ is the smallest torsion class containing $\alpha(\mathcal{T})$, it is enough to check that $\mathcal{T} \subseteq \mathcal{T}_{\alpha(\mathcal{T})}$. We take a non-zero module $X$ in $\mathcal{T}$. By induction, it suffices to show that $\operatorname{Hom}_{A}(\alpha(\mathcal{T}), X) \neq 0$. Suppose that the opposite holds. Let $\phi: A \rightarrow T_{0}$ be the minimal left $\mathcal{T}$ approximation of the regular module. In what follows, we view $\operatorname{Hom}_{A}\left(T_{0}, X\right)$ as a right $\operatorname{End}_{A}\left(T_{0}\right)$ module. Note that $\operatorname{Hom}_{A}\left(T_{0}, X\right) \neq 0$, since $X$ is in $\mathcal{T}$. Let $S$ be a simple submodule of $\operatorname{Hom}_{A}\left(T_{0}, X\right)$ such that $S=f \cdot \operatorname{End}_{A}\left(T_{0}\right)$ for a morphism $f: T_{0} \rightarrow X$. By $t_{T_{1}}\left(T_{0}\right)$ we denote the $A$-module trace of $T_{1}$ in $T_{0}$. Using Lemma 3.8, it follows that $T_{0} / t_{T_{1}}\left(T_{0}\right)$ lies in $\alpha(\mathcal{T})$ and, thus, by assumption, all maps from $T_{0} / t_{T_{1}}\left(T_{0}\right)$ to $X$ are zero. Consequently, the $A$-module $t_{T_{1}}\left(T_{0}\right)$ cannot be contained in the kernel of $f$. Therefore, there is a map $g: T_{1} \rightarrow T_{0}$ such that $f \circ g: T_{1} \rightarrow X$ is not zero. Moreover, since $T_{0}$ generates $\mathcal{T}$, we also obtain a map $h: T_{0} \rightarrow T_{1}$ such that the composition

$$
T_{0} \stackrel{h}{\longrightarrow} T_{1} \stackrel{g}{\longrightarrow} T_{0} \stackrel{f}{\longrightarrow} X
$$

is not trivial. But, using Lemma 3.7, the map $g \circ h$ belongs to the radical of $E n d_{A}\left(T_{0}\right)$ and, thus, the composition $f \circ g \circ h$ lies in $S \cdot \operatorname{rad}\left(\operatorname{End}_{A}\left(T_{0}\right)\right)=\operatorname{rad}(S)=0$. This yields a contradiction.

We summarise the results above in the following theorem.

Theorem 3.10. Let $A$ be a finite dimensional $\mathbb{K}$-algebra. Then $\alpha$ yields a bijective correspondence between

(1) functorially finite torsion classes in $\bmod (A)$;

(2) functorially finite wide subcategories $\mathcal{W}$ in $\bmod (A)$ for which $\mathcal{T}_{\mathcal{W}}$ is functorially finite.

Proof. This follows from Proposition 3.3 and Proposition 3.9.

Note that, in general, the assumption above of $\mathcal{T}_{\mathcal{W}}$ being functorially finite is not automatic (see Lemma 3.12 and Example 4.7). Nevertheless, it can be dropped in some contexts. 
Corollary 3.11. Let $A$ be a finite dimensional $\mathbb{K}$-algebra with $\mid f$-tors $(A) \mid<\infty$. Then $\alpha$ yields a bijection

$$
\operatorname{tors}(A) \longrightarrow \text { wide }(A) \text {. }
$$

Moreover, all torsion classes and all wide subcategories in $\bmod (A)$ are functorially finite.

Proof. Using [DIJ, Theorem 3.8], $\mid f$-tors $(A) \mid<\infty$ implies that all torsion classes in $\bmod (A)$ are functorially finite. Now the statement follows from Theorem 3.10. In fact, all wide subcategories of $\bmod (A)$ are functorially finite, by Proposition 3.3 and Lemma 3.8

This corollary, in particular, applies to representation finite $\mathbb{K}$-algebras. Furthermore, examples of representation infinite algebras with only finitely many torsion classes can be found in $[\mathrm{A}]$ and [Mi], namely certain Brauer graph algebras and preprojective algebras of Dynkin type.

In some special cases, similar bijections to the ones above were discussed in the literature before. If $A$ is a hereditary algebra, it was proven in [IT, Corollary 2.17] that $\alpha$ yields a bijection between functorially finite torsion classes and functorially finite wide subcategories in $\bmod (A)$. Here, $\mathcal{T}_{\mathcal{W}}$ is given by $\operatorname{gen}(\mathcal{W})$. Moreover, it follows from [M], Proposition 6.2] that $\alpha$ induces a similar bijection for Nakayama algebras with $\mathcal{T}_{\mathcal{W}}$ being

$$
\operatorname{gen}(\mathcal{W}) \star \operatorname{gen}(\mathcal{W}):=\{X \in \bmod (A) \mid \exists 0 \rightarrow M \rightarrow X \rightarrow N \rightarrow 0, M, N \in \operatorname{gen}(\mathcal{W})\}
$$

We finish this section by discussing necessary and sufficient conditions for the torsion class $\mathcal{T}_{\mathcal{W}}$ to be functorially finite for $\mathcal{W} \in f$-wide $(A)$. Let $\operatorname{gen}(\mathcal{W})^{\star n}$ be the class of modules possessing a $\operatorname{gen}(\mathcal{W})$-filtration of length at most $n$ for $n \in \mathbb{N}$.

Lemma 3.12. The following are equivalent for $\mathcal{W}$ in $f$-wide $(A)$ :

(1) $\mathcal{T}_{\mathcal{W}}$ is functorially finite.

(2) There exists $n \geq 1$ such that $\mathcal{T}_{\mathcal{W}}=\operatorname{gen}(\mathcal{W})^{\star n}$.

Proof. (1) $\Rightarrow(2)$. Let $\phi: A \rightarrow T_{0}$ be the minimal left $\mathcal{T}_{\mathcal{W}}$-approximation of $A$. Then certainly $T_{0} \in \operatorname{gen}(\mathcal{W})^{\star n}$ for some $n \geq 1$, since $\mathcal{T}_{\mathcal{W}}=\bigcup_{n \geq 1} \operatorname{gen}(\mathcal{W})^{\star n}$. Now given any $M \in \mathcal{T}_{\mathcal{W}}$ and a

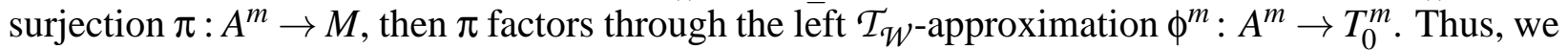
have a surjection $T_{0}^{m} \rightarrow M$, proving that $M \in \operatorname{gen}(\mathcal{W})^{\star n}$.

$(2) \Rightarrow(1)$. Since $\mathcal{W}$ is functorially finite, so are $\operatorname{gen}(\mathcal{W})$ (see $[\mathrm{AS}, \S 4])$ and $\mathcal{T}_{\mathcal{W}}=\operatorname{gen}(\mathcal{W})^{\star n}$ (compare, for example, to [GT, Corollary 1.3]).

\section{UNIVERSAL LOCALISATIONS}

Classifying functorially finite wide subcategories of $\bmod (A)$ at the same time translates to classifying certain ring epimorphisms starting in $A$ (up to equivalence). Let us be a bit more precise. A ring epimorphism is an epimorphism in the category of all (unital) rings. It is well-known that ring epimorphisms describe precisely those ring homomorphisms for which restriction yields a fully faithful functor between the corresponding module categories. We say that two ring epimorphisms $f: A \rightarrow B$ and $g: A \rightarrow C$ are equivalent if there is a (necessarily unique) isomorphism of rings $h: B \rightarrow C$ such that $g=h \circ f$. Moreover, for a given ring epimorphism $A \rightarrow B$, it was proven in [SCh, Theorem 4.8] that $\operatorname{Tor}_{1}^{A}(B, B)=0$ if and only if $\operatorname{Ext}_{A}^{1}(M, N) \cong \operatorname{Ext}_{B}^{1}(M, N)$ for all $B$-modules $M$ and $N$. The following proposition, essentially due to [GL] (also compare to [I, Theorem 1.6.1]), is important in our context.

Proposition 4.1. Let $A$ be a finite dimensional $\mathbb{K}$-algebra. By assigning to a given ring epimorphism the essential image of its associated restriction functor, one gets a bijection between 
(1) equivalence classes of ring epimorphisms $A \rightarrow B$ with $\operatorname{dim}_{\mathbb{K}}(B)<\infty$ and $\operatorname{Tor}_{1}^{A}(B, B)=0$;

(2) functorially finite wide subcategories of $\bmod (A)$.

Using our results obtained in the previous section, we can describe many of these ring epimorphisms more explicitly by using the concept of universal localisation.

Theorem 4.2. [Sch, Theorem 4.1] Let $\Sigma$ be a set of maps between finitely generated projective $A$-modules. Then there is a ring $A_{\Sigma}$, the universal localisation of $A$ at $\Sigma$, and a ring homomorphism $f: A \rightarrow A_{\Sigma}$ such that

(1) $A_{\Sigma} \otimes_{A} \sigma$ is an isomorphism for all $\sigma \in \Sigma$;

(2) every ring homomorphism $g: A \rightarrow B$ such that $B \otimes_{A} \sigma$ is an isomorphism for all $\sigma \in \Sigma$ factors in a unique way through $f$, i.e. there is a commutative diagram of the form

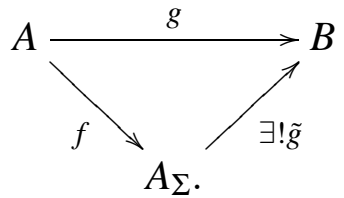

Moreover, $f: A \rightarrow A_{\Sigma}$ is a ring epimorphism fulfilling $\operatorname{Tor}_{1}^{A}\left(A_{\Sigma}, A_{\Sigma}\right)=0$.

Note that, in general, the $\mathbb{K}$-dimension of $A_{\Sigma}$ can be infinite. If it is finite, we can consider the restriction functor $f_{*}: \bmod \left(A_{\Sigma}\right) \rightarrow \bmod (A)$ whose essential image is given by those $X \in \bmod (A)$ for which $\operatorname{Hom}_{A}(\sigma, X)$ is an isomorphism for all $\sigma \in \Sigma$. The philosophy is that most (and conjecturally all) of the ring epimorphisms arising from functorially finite wide subcategories of $\bmod (A)$ are universal localisations. To prove this, we continue to study in more detail the objects in $f$-tors $(A)$.

Let $\mathcal{T}=\operatorname{gen}(T)$ be a functorially finite torsion class in $\bmod (A)$ with respect to the silting module $T$ and its projective presentation $\sigma$. Then $\mathcal{T}=\mathcal{D}_{\sigma}=\left\{X \in \bmod (A) \mid \operatorname{Hom}_{A}(\sigma, X)\right.$ surjective $\}$ (compare to Definition 3.5). Consider the $\mathcal{T}$-approximation sequence

$$
A \stackrel{\phi}{\longrightarrow} T_{0} \longrightarrow T_{1} \longrightarrow 0
$$

with $T_{0}$ and $T_{1}$ in $\operatorname{add}(T)$ and $\phi$ left-minimal. Note that $T^{\prime}:=T_{0} \oplus T_{1}$ is a silting module equivalent to $T$, i.e. $\operatorname{add}(T)=\operatorname{add}\left(T^{\prime}\right)$. In fact, if we choose the projective presentation $\sigma^{\prime}$ of $T^{\prime}$ to be the direct sum of the minimal projective presentation of $T^{\prime}$ and the trivial map $A e \rightarrow 0$ for an idempotent $e \in A$, that is chosen maximal such that $\operatorname{Hom}_{A}(A e, T)=0$, then also $\mathcal{D}_{\sigma}=\mathcal{D}_{\sigma^{\prime}}$. Similar to the situation for tilting modules one also recovers $\mathcal{T}$ from $T_{1}$.

Lemma 4.3. With the above notation, there is a projective presentation $\sigma_{1}$ of $T_{1}$ such that $\mathcal{D}_{\sigma_{1}}=\mathcal{T}$ (in particular, $T_{1}$ is a partial silting module with respect to $\sigma_{1}$ following [AMV, Definition 3.10]).

Proof. Let $\sigma_{0}^{\prime} \in \operatorname{add}(\sigma)$ be the minimal projective presentation of the module $T_{0}$ from (4.1). We will view $\sigma_{0}^{\prime}$ as a two-term complex in the derived category $D(A):=D(\bmod (A))$. There the approximation map $\phi: A \rightarrow T_{0}$ factors through the cokernel map $\pi: \sigma_{0}^{\prime} \rightarrow T_{0}$. That is, there is a map $\psi: A \rightarrow \sigma_{0}^{\prime}$ in $D(A)$ such that $\phi=\pi \circ \psi$ and, in particular, $\operatorname{Hom}_{D(A)}(\psi, T)$ is surjective. Now consider the triangle

$$
A \stackrel{\psi}{\longrightarrow} \sigma_{0}^{\prime} \longrightarrow \sigma_{1}^{\prime} \longrightarrow A[1] .
$$

Then $\sigma_{1}^{\prime}$ is also a two-term complex of finitely generated projective $A$-modules and $\operatorname{coker}\left(\sigma_{1}^{\prime}\right)=T_{1}$. A priori, we do not know whether $\sigma_{1}^{\prime}$ is a minimal presentation of $T_{1}$. However, an application of 
the functor $\operatorname{Hom}_{D(A)}(-, T[1])$ yields an exact sequence

$$
\operatorname{Hom}_{D(A)}\left(\sigma_{0}^{\prime}[1], T[1]\right) \stackrel{\psi^{*}}{\longrightarrow} \operatorname{Hom}_{D(A)}(A[1], T[1]) \longrightarrow \operatorname{Hom}_{D(A)}\left(\sigma_{1}^{\prime}, T[1]\right) \longrightarrow \operatorname{Hom}_{D(A)}\left(\sigma_{0}^{\prime}, T[1]\right)
$$

and, since $T \in \mathcal{D}_{\sigma}$ and $\operatorname{Hom}_{D(A)}(\psi, T)$ is surjective, it follows that $\operatorname{Hom}_{A}\left(\sigma_{1}^{\prime}, T\right)$ is surjective. Thus, any potential summand $A e^{\prime} \rightarrow 0$ of $\sigma_{1}^{\prime}$ is such that $\operatorname{Hom}_{A}\left(A e^{\prime}, T\right)=0$.

Let again $e \in A$ be a maximal idempotent such that $\operatorname{Hom}_{A}(A e, T)=0$ and let $\sigma_{1}$ be the map obtained from $\sigma_{1}^{\prime}$ by adding $A e \rightarrow 0$. Then we have shown that the complex $\sigma_{1}$ lies in add $(\sigma)$ and hence $\mathcal{D}_{\sigma} \subseteq \mathcal{D}_{\sigma_{1}}$. For the inclusion $\mathcal{D}_{\sigma_{1}} \subseteq \mathcal{D}_{\sigma}$, take a module $M$ in $\mathcal{D}_{\sigma_{1}}$ and apply the functor $\operatorname{Hom}_{D(A)}(-, M)$ to the triangle in (4.2). We learn that $\operatorname{Hom}_{A}\left(\sigma_{0}^{\prime}, M\right)$ is surjective and since $\operatorname{add}(\sigma)=\operatorname{add}\left(\sigma_{0}^{\prime} \oplus \sigma_{1}\right)$ by construction, necessarily $M \in \mathcal{D}_{\sigma}$.

Next, we provide sufficient conditions for a ring epimorphism to be a universal localisation.

Proposition 4.4. Let $A \rightarrow B$ be a ring epimorphism with $\operatorname{dim}_{\mathbb{K}}(B)<\infty$ and $\operatorname{Tor}_{1}^{A}(B, B)=0$. By $X_{B}$ we denote the essential image of the restriction functor in $\bmod (A)$. If $\mathcal{T}_{X_{B}}=$ filt $\left(\operatorname{gen}\left(X_{B}\right)\right)$ is functorially finite, then $B$ is the universal localisation of $A$ at a map $\sigma_{B}$ between finitely generated projective A-modules with $\operatorname{Hom}_{D(A)}\left(\sigma_{B}, \sigma_{B}[1]\right)=0$.

Proof. Since, by assumption, $\mathcal{T}_{X_{B}}$ is functorially finite, it is of the form $\operatorname{gen}(T)$ for some finite dimensional silting $A$-module $T$. Now consider the $\operatorname{gen}(T)$-approximation sequence

$$
A \stackrel{\phi}{\longrightarrow} T_{0} \longrightarrow T_{1} \longrightarrow 0
$$

with $\phi$ left-minimal and $T_{0}$ and $T_{1}$ in $\operatorname{add}(T)$. By Lemma 4.3 , there is a projective presentation $\sigma_{1}$ of $T_{1}$ turning $T_{1}$ into a partial silting module with

$$
\operatorname{gen}(T)=\mathcal{D}_{\sigma_{1}}=\left\{X \in \bmod (A) \mid \operatorname{Hom}_{A}\left(\sigma_{1}, X\right) \text { is surjective }\right\} .
$$

We define $\sigma_{B}:=\sigma_{1}$. Using Proposition 3.3 and Lemma 3.8 , it follows that

$$
X_{B}=\alpha(\operatorname{gen}(T))=\operatorname{gen}(T) \cap T_{1}^{\circ}=\left\{X \in \bmod (A) \mid \operatorname{Hom}_{A}\left(\sigma_{1}, X\right) \text { is an isomorphism }\right\} .
$$

It remains to check that the universal localisation of $A$ at $\left\{\sigma_{1}\right\}$ is finite dimensional. But this is a direct consequence of the construction in [AMV2]. In fact, there is an isomorphism of algebras

$$
A_{\left\{\sigma_{1}\right\}} \cong \operatorname{End}_{A}^{o p}\left(T_{0} \oplus T_{1}\right) /\left\langle e_{T_{1}}\right\rangle
$$

where $e_{T_{1}}$ denotes the idempotent in $E n d_{A}^{o p}\left(T_{0} \oplus T_{1}\right)$ associated to the summand $T_{1}$.

In the context of representation finite algebras, we obtain the following theorem that generalises previous work on Nakayama algebras (see [M, Corollary 6.3]).

Theorem 4.5. Let $A$ be a finite dimensional and representation finite $\mathbb{K}$-algebra. Then the following sets are in bijection:

(1) isomorphism classes of basic silting modules in $\bmod (A)$;

(2) torsion classes in $\bmod (A)$;

(3) wide subcategories of $\bmod (A)$;

(4) epiclasses of ring epimorphisms $A \rightarrow B$ with $\operatorname{Tor}_{1}^{A}(B, B)=0$;

(5) epiclasses of universal localisations of $A$.

In particular, for all universal localisations $A_{\Sigma}$ of $A$ there is a map $\sigma$ between finitely generated projective A-modules with $\operatorname{Hom}_{D(A)}(\sigma, \sigma[1])=0$ such that $A_{\Sigma}=A_{\{\sigma\}}$. 
Proof. The bijection (1) $\Leftrightarrow(2)$ is due to [AIR, Theorem 2.7]. Moreover, the correspondence $(2) \Leftrightarrow(3)$ follows from Corollary 3.11. The bijection $(3) \Leftrightarrow(4)$ is exactly Proposition 4.1. To see this, recall that for representation finite algebras, all ring epimorphisms $A \rightarrow B$ have a finite dimensional codomain ([GdP Corollary 2.3]). Finally, the bijection $(4) \Leftrightarrow(5)$ is a consequence of Proposition 4.4

Example 4.6. Let us consider the situation from [AIR, Example 6.4] and [M, Examples 5.5 and 6.6], i.e. the algebra $A=\mathbb{K} Q /\langle\beta \alpha\rangle$ with the quiver

$$
Q: 1 \stackrel{\alpha}{\longrightarrow} 2 \stackrel{\beta}{\longrightarrow} 3 .
$$

Then $T=S_{1} \oplus P_{1} \oplus P_{3}$ is a sincere $\tau$-tilting module which is not tilting.

Let us compute the corresponding universal localisation. We simply have $\mathcal{T}=\operatorname{gen}(T)=\operatorname{add}(T)$ and the minimal $\mathcal{T}$-approximation sequence for $P_{2}$ is

$$
P_{2} \stackrel{-\cdot \alpha}{\longrightarrow} P_{1} \longrightarrow S_{1} \longrightarrow 0
$$

which is at the same time the minimal projective presentation for $S_{1}$. Hence, the minimal approximation sequence (4.1) for $A$ is of the form $A \rightarrow P_{1} \oplus P_{1} \oplus P_{3} \rightarrow S_{1} \rightarrow 0$. It follows that $P_{1}, P_{3}$ are precisely the indecomposable split projective modules in $\mathcal{T}$ while $S_{1}$ is the only indecomposable Ext-projective module which is not split projective. The corresponding universal localisation $f$ is obtained by inverting the minimal projective presentation of $S_{1}$, or in other words, we adjoin an inverse $\alpha^{-1}$ to $\alpha$ in $A$. Clearly, $f: A \rightarrow A_{\{\alpha\}}$ cannot be injective since $\beta \cdot T=0$, and indeed one easily checks that $\operatorname{ker}(f)=\langle\beta\rangle$. As a ring, $A_{\{\alpha\}} \cong \operatorname{End}_{A}^{o p}\left(P_{1} \oplus P_{1} \oplus P_{3} \oplus S_{1}\right) /\left\langle e_{S_{1}}\right\rangle \cong M_{2}(\mathbb{K}) \times \mathbb{K}$.

Combining [AIR, Example 6.4] with Theorem 4.5, there are precisely 12 universal localisations up to equivalence originating at our $A$. Similar easy computations can be performed for a range of other representation finite algebras whose representation theory is sufficiently well understood; see e.g. [AIR, §6] or [M].

The following example was communicated to us by Sota Asai, see [Asai, Example 4.5].

Example 4.7. Consider the algebra $A=\mathbb{K} Q /\langle\gamma \alpha\rangle$ with the quiver

$$
Q: 1 \underset{\beta}{\stackrel{\alpha}{\longrightarrow}} 2 \stackrel{\gamma}{\longrightarrow} 3 \text {. }
$$

Note that $A$ is a tilted algebra of type $\widetilde{A}_{2}$. Since the indecomposable injective $A$-module $I_{3}$ has no non-trivial endomorphisms, it follows that $\mathcal{W}=\operatorname{add}\left(I_{3}\right)$ is a functorially finite wide subcategory of $\bmod (A)$. It was shown by Asai that the torsion class $\mathcal{T}_{\mathcal{W}}$ is not functorially finite. Indeed, otherwise also the intersection $\mathcal{T}_{\mathcal{W}} \cap P_{3}^{\circ}=\operatorname{filt}\left(\operatorname{gen}\left(I_{3} / S_{3}\right)\right)$ would be functorially finite in $X_{A / A e_{3} A} \cong \bmod \left(A / A e_{3} A\right)$, yielding a contradiction, since $I_{3} / S_{3}$ identifies with a simple regular module over the Kronecker algebra $A / A e_{3} A$. In particular, it follows that the assignment $\alpha$ from Theorem 3.10 only yields an injective map $f$-tors $(A) \rightarrow f$-wide $(A)$ and not a bijection (see Asai, Theorem 4.6] for when $f$-tors $(A) \rightarrow f$-wide $(A)$ is a bijection for other tilted algebras $A$ ).

Nevertheless, it turns out that the functorially finite wide subcategory $\mathcal{W}$ coincides with the essential image of the restriction functor of the universal localisation of $A$ at the map

$$
\sigma:=\left(P_{3} \stackrel{-\cdot \gamma}{\longrightarrow} P_{2}\right) \oplus\left(P_{3} \stackrel{-\cdot \gamma \beta}{\longrightarrow} P_{1}\right) .
$$


Indeed, on the one hand, $I_{3}$ clearly carries an $A_{\{\sigma\}}$-module structure and, on the other hand, by adjoining inverses $\gamma^{-1}$ and $(\gamma \beta)^{-1}$ to $\gamma$ and $\gamma \beta$ in $A$ we obtain the localisation $A_{\{\sigma\}}$ which is Morita equivalent to $\mathbb{K}$. Hence, we have $\mathcal{W}=X_{A_{\{\sigma\}}}$. Moreover, it is a straightforward computation to check that $\operatorname{Hom}_{D(A)}(\sigma, \sigma[1])=0$. It follows that not all universal localisations of $A$ at such a map $\sigma$ arise from the process described in Proposition 4.4 and Example 4.6. In other words, different techniques are needed if one hopes to prove a general version of Theorem 4.5 relating silting modules to universal localisations.

\section{REFERENCES}

[A] Adachi, T.: Characterizing $\tau$-tilting finite algebras with radical square zero, Proc. Amer. Math. Soc. 144 (2016), 4673-4685.

[AA] Angeleri Hügel, L.; Archetti, M.: Tilting modules and universal localization, Forum Math. 24 (2012), no. 4 , 709-731.

[AHK] Angeleri Hügel, L.; Happel, D.; Krause, H.: Handbook of tilting theory, London Math. Soc. Lecture Note Ser. 332, Cambridge Univ. Press, Cambridge, 2007.

[AIR] Adachi, T.; Iyama, O.; Reiten, I.: $\tau$-tilting theory, Compos. Math. 150 (2014), 415-452.

[AMV] Angeleri Hügel, L.; Marks, F.; Vitória, J.: Silting modules, Int. Math. Res. Not. IMRN 2016, no. 4, 12511284.

[AMV2] Angeleri Hügel, L.; Marks, F.; Vitória, J.: Silting modules and ring epimorphisms, Adv. Math. 303 (2016), 1044-1076.

[Asai] Asai, S.: Semibricks, preprint, arXiv:1610.05860v1.

[AS] Auslander, M.; Smal $\varnothing$, S.O.: Preprojective modules over artin algebras, J. Algebra 66 (1980), no. 1, 61-122.

[AS2] Auslander, M.; Smalø, S.O.: Almost split sequences in subcategories, J. Algebra 69 (1981), no. 2, 426-454.

[D] Dickson, S.E.: A torsion theory for abelian categories, Trans. Amer. Math. Soc. 121 (1966), 223-235.

[DIJ] Demonet, L.; Iyama, O.; Jasso, G.: $\tau$-tilting finite algebras, $g$-vectors and brick- $\tau$-rigid correspondence, preprint, arXiv:1503.00285v5

[GdP] Gabriel, P.; de la Peña, J.A.: Quotients of representation-finite algebras, Comm. Algebra 15 (1987), no. 1-2, 279-307.

[GL] Geigle, W.; Lenzing, H.: Perpendicular categories with applications to representations and sheaves, J. Algebra 144 (1991), no. 2, 273-343.

[GT] Gentle, R.; Todorov, G.: Extensions, kernels and cokernels of homologically finite subcategories, Representation theory of algebras, CMS Conf. Proc. 18, Amer. Math. Soc., Providence, RI, 1996, 227-235.

$[\mathrm{H}] \quad$ Hovey, M.: Classifying subcategories of modules, Trans. Amer. Math. Soc. 353 (2001), no. 8, 3181-3191.

[Hop] Hopkins, M.J.: Global methods in homotopy theory, Homotopy theory (Durham, 1985) (J. D. S. Jones and E. Rees, eds.), London Math. Soc. Lecture Note Ser. 117, Cambridge Univ. Press, Cambridge, 1987, 73-96.

[I] Iyama, O.: Rejective subcategories of artin algebras and orders, preprint, arXiv:math/0311281.

[IT] Ingalls, C.; Thomas, H.: Noncrossing partitions and representations of quivers, Compos. Math. 145 (2009), 1533-1562.

[KS] Kashiwara, M.; Schapira, P.: Categories and sheaves, Grundlehren der Mathematischen Wissenschaften 332, Springer-Verlag, Berlin, 2006.

[M] Marks, F.: Universal localisations and tilting modules for finite dimensional algebras, J. Pure Appl. Algebra 219 (2015), 3053-3088.

[Mi] Mizuno, Y.: Classifying $\tau$-tilting modules over preprojective algebras of Dynkin type, Math. Z. 277 (2014), no. 3, 665-690.

[N] Neeman, A.: The chromatic tower for D(R), Topology 31 (1992), 519-532.

[NR] Neeman, A.; Ranicki, A.: Noncommutative localisation in algebraic K-theory I, Geometry and Topology 8 (2004), 1385-1425.

[R] Ranicki, A.: Noncommutative localization in topology, Noncommutative localization in algebra and topology, London Math. Soc. Lecture Note Ser. 330, Cambridge Univ. Press, Cambridge, 2006, 81-102.

[Sch] Schofield, A.: Representations of rings over skew fields, London Math. Soc. Lecture Note Ser. 92, Cambridge Univ. Press, Cambridge, 1985. 
[Sm] Smalø, S.O.: Torsion theories and tilting modules, Bull. Lond. Math. Soc. 16 (1984), no. 5, 518-522.

[T] Thomason, R.W.: The classification of triangulated subcategories, Compos. Math. 105 (1997), no. 1, 1-27.

Frederik Marks, University of Stuttgart, Institute for Algebra and Number Theory, PfafFENWALDRING 57, 70569 STUTTGART, GERMANY

E-mail address: marks@mathematik.uni-stuttgart.de

Jan ŠŤovíček, Charles University in Prague, Faculty of Mathematics and Physics, Department of Algebra, Sokolovská 83, 18675 Praha, Czech Republic

E-mail address: stovicek@karlin.mff.cuni.cz 